\title{
Flexible word classes in linguistic typology and grammatical theory
}

\author{
EVA VAN LIER AND JAN RIJKHOFF
}

\subsection{Introduction}

Currently one of the most controversial topics in linguistic typology and grammatical theory concerns the existence of fLEXIBLE LANGUAGES, i.e. languages with a word class whose members cover functions that are typically associated with two or more of the traditional word classes (verb, noun, and adjective). ${ }^{1,2}$ This anthology aims to broaden the empirical and theoretical horizon of the discussion surrounding such flexible languages and to make a significant contribution to our understanding of lexical flexibility in human language.

The present volume presents an up-to-date reflection of approximately two decades of cross-linguistic and theoretical research on flexible parts of speech. It contains substantially elaborated versions of some of the papers presented at the international workshop on flexible languages, held at the University of Amsterdam in 2007. This concerns the papers by Jan Don and Eva van Lier, David Gil, Kees Hengeveld, Sebastian Nordhoff, and John Peterson. In addition, the present volume contains invited contributions on flexibility in part of speech systems by David Beck, Walter Bisang, William McGregor, and Felix Rau.

\footnotetext{
${ }^{1}$ We use the phrase 'traditional word class' (verb, noun, adjective, etc.) for lexical categories with a functional core, but defined by language-specific distributional properties, following the classical European (Aristotle, Dyonisius Thrax) and American structuralist tradition (Franz Boas). Notice that adjectives have only been regarded as a separate word class since the second half of the 18th century (Priestley 1761, Beauzée 1767). As is common in the typological literature, we capitalise language-specific categories. Notably, we do not regard the 'traditional word classes' as universal linguistic categories, as is the norm in the generativist framework.

${ }^{2}$ Unless explicitly stated otherwise, we use the term 'word' in the sense of 'lexeme' or 'root', meaning that (at this point) we are not interested in morphologically derived and/or inflected words. A 'word class', then, is a (language-specifically defined) grouping of lexemes or roots. 'Word class' is used interchangeably with 'part of speech'. A 'parts-of-speech system' refers to the set of major word classes in a particular language.
} 
We should make explicit that this volume does not aim to integrate the individual chapters under a single theoretical approach. It rather presents a wide range of novel descriptive facts and shows how some grammatical theories could accommodate these facts. ${ }^{3}$ Some of the chapters are primarily theoretically and typologically oriented, taking into account several languages (see especially the contribution by Hengeveld; also those by Don and van Lier; Bisang). Other chapters combine a theoretical analysis with the description of flexibility in a single language (chapters by Gil, Peterson, and McGregor). Finally, a number of case studies of individual languages illustrate variation in the realm of lexical flexibility and discuss the questions this raises for the typology and diachrony of lexical flexibility (chapters by Rau, Beck, Nordhoff, and Bisang).

This introductory chapter is organized as follows. In Section 1.2 we set the scene with a discussion of some general problems of categorization, in particular (i) cases where one entity seems to belong to two or more categories, and (ii) cases where entities appear to be category neutral or underspecified. It will be shown that the solutions for these categorization problems that have been proposed in the area of linguistics are basically identical to the kind of solutions that have been proposed in other sciences like biology or astronomy. Section 1.3 provides an overview of functional-typological research on flexibility in part of speech systems. The section begins with a general introduction to the main developments in this area, followed by a discussion of the set of criteria to establish lexical flexibility as proposed by Evans and Osada (2005). In Section 1.4, we consider flexibility from a wider perspective, going beyond the domain of lexical categorization. Several recent studies (including some contained in the present volume) suggest that (a certain degree of) flexibility in the parts-of-speech system of a language can be related to (different degrees of) flexibility-or rather the lack of it-at other levels of the grammar of that language. Subsequently we sketch a proposal for a new, four-way typology of flexibility, covering both lexical and grammatical linguistic categories. Section 1.5, finally, provides an overview of the contents of this book.

\subsection{Categorization: approaches and problems}

\subsubsection{To cognize is to categorize}

Categorization can be regarded as one of the most fundamental traits of human cognition ('to cognize is to categorize'-cf. Harnad 2005): putting people or things, but also more abstract entities such as words, into groups on the basis of certain

\footnotetext{
${ }^{3}$ Most generative theoretical frameworks have denied the existence of lexical flexibility in individual languages (for a recent example, see Chung 2012). The Minimalist-based theory of Distributed Morphology, however, assumes that the lexical material of any language is uncategorized; a lexeme is categorized by combining it with a syntactic functional head (Halle and Marantz 1993; Marantz 1997).
} 
shared characteristics. Currently two main approaches to categorization can be distinguished: CLASSICAL or LOGICAL CATEGORIZATION and PROTOTYPE THEORY (for overviews, see e.g. Aarts 2006; van der Auwera and Gast 2011). ${ }^{4}$ In classical categorization, each object is deemed to belong to a single (discrete, unique) category, whose members all share the same set of necessary and sufficient properties. In prototype theory, by contrast, it is not assumed that all members of a category have exactly the same set of properties (e.g. Rosch 1975, 1983). Here categorization is rather based on prototypes, allowing for more and less typical class members. For example, a bird that can fly is a better, more representative member of the category BIRD than a flightless bird like a penguin or a kiwi. ${ }^{5}$

However, since both approaches recognize that categories have (either fuzzy or sharp) boundaries (without a boundary a category is essentially useless; cf. Cruse 2004: 135), they face the same problem: what should be done with entities that do not seem to fall within the boundary of a single, existing category? This problem mainly arises for one of two reasons: (i) an entity has properties that are characteristic for objects that belong to two or more distinct categories, or (ii) an entity has properties that are not characteristic for any one of the established categories, even though there may be a (developmental or functional) connection with some of the established categories.

(i) Classification of objects that seem to belong to different categories A well-known example of an object that seems to belong to various categories comes from biology and concerns fungi (a large group of organisms that includes mushrooms, but also microorganisms like yeasts and moulds), which have properties of both plants and animals. Before the introduction of molecular methods for phylogenetic analysis, fungi were regarded as plants, but they are now classified as a separate kingdom, distinct from plants, animals, and bacteria. To give an example from another domain: as a consequence of the recent redefinition of 'planet', there is now a new category of astronomical objects called 'plutoid planets', which combines certain characteristics of two other categories: dwarf planets and trans-Neptunian objects. These examples show how new categories are added to the set of established categories in order to accommodate entities that do not belong to an existing category.

(ii) Classification of underspecified objects There are also objects that are difficult to fit into one of the conventional categories because they are underspecified, i.e. characterized by multifunctional behaviour or rather the potential to develop into

\footnotetext{
${ }^{4}$ We will ignore conceptual clustering, an approach that has been developed in the context of machine learning (see e.g. Stepp and Michalski 1986; Fisher and Pazzani 1991).

5 The model developed by Aarts (2004 and later work) is probably the most comprehensive contemporary prototype theory of word classes. One of the problems with his model is that it is based on English word classes. For further critical assessment of Aarts's theory, see Croft (2007) and Smith (2011).
} 
various more specific types. In cell biology, for example, stem cells have the ability to differentiate into more specialized cell types. Thus, totipotent cells have the potential to divide and produce all the differentiated cells in an organism, whereas pluripotent stem cells can become any tissue in the body, except placenta.

This type of category indeterminacy can be regarded as temporary. It implies the existence of one or more subsequent developmental stages that are characterized by a (gradual) loss of flexibility, and an increase of categorial specificity.

\subsubsection{Categorization of flexible words}

Linguistics is no different from the other sciences when it comes to categorization issues, and solutions similar to the ones outlined in Section 1.2.1 have been proposed for problems in determining word class membership. Thus we see that flexible words, which fall outside the boundaries of the traditional word classes, are either regarded as members of a new category, or given the special status of precategorial or categoryneutral items.

(i) Flexible words as members of a new word class We saw in Section 1.2.1 that we sometimes encounter a group of objects with properties that are characteristic for members of different established categories. Thus, fungi have properties of both animals and plants, but since the characteristic properties of fungi cannot be reduced to the typical properties of either one of these traditional categories, they are now classified as a separate category. Basically the same argument has been made in the case of flexible words, which seem to occur at the intersection of traditional word classes like verbs, nouns and adjectives. Since the characteristic properties of flexible words cannot be reduced to the properties of just one of these traditional word classes, they should form categories of their own. This is the approach taken in Hengeveld's cross-linguistic classification of parts-of-speech (PoS) systems, shown in Figure 1.1, which distinguishes three types of flexible word classes: 'contentives', 'non-verbs', and 'modifiers'.

Hengeveld's proposal is discussed in more detail in Section 1.3.1, but it is important to point out here that, even though from a functional perspective, members of a flexible word class occur at the intersection of two or more traditional word classes, flexible word classes are not the product of a merge process. ${ }^{6,7}$ This probably also explains why members of a flexible word class do not have the combined (accumulated) characteristic lexical properties of traditional word classes (Rijkhoff 2008a: 240-1). Thus,

\footnotetext{
6 This view is apparently not shared by Evans and Osada (2005: 385), who characterize a flexible word class as 'a single merged class'.

${ }^{7}$ Notably, considering flexible categories as non-merged does not exclude the possibility that, in the course of time, a functionally differentiated word class may develop out of a flexible word class (cf. Hurford 2007). Smit (2001) shows how derivational processes play a central role in this diachronic development (see also Nordhoff this volume).
} 


\begin{tabular}{|c|c|c|c|c|c|}
\hline \multicolumn{2}{|l|}{$\begin{array}{l}\text { PoS } \\
\text { system }\end{array}$} & $\begin{array}{l}\text { Head of } \\
\text { predicate } \\
\text { phrase }\end{array}$ & $\begin{array}{l}\text { Head of } \\
\text { referential } \\
\text { phrase }\end{array}$ & $\begin{array}{l}\text { Modifier of } \\
\text { head of referential } \\
\text { phrase }\end{array}$ & $\begin{array}{l}\text { Modifier of } \\
\text { head of predicate } \\
\text { phrase }\end{array}$ \\
\hline \multirow{3}{*}{ Flexible } & 1 & \multicolumn{4}{|c|}{ CONTENTIVE } \\
\hline & 2 & Verb & \multicolumn{3}{|c|}{ NON-VERB } \\
\hline & 3 & Verb & Noun & \multicolumn{2}{|c|}{ MODIFIER } \\
\hline Differentiated & 4 & Verb & Noun & Adjective & Manner Adverb \\
\hline \multirow{3}{*}{ Rigid } & 5 & Verb & Noun & Adjective & - \\
\hline & 6 & Verb & Noun & - & - \\
\hline & 7 & Verb & - & - & - \\
\hline
\end{tabular}

FIGURE 1.1 Part of speech systems with flexible word classes in small caps (based on Hengeveld this volume)

a flexible word class like Hengeveld's contentive is a distinct lexical category in its own right, with its own unique set of lexical features. Perhaps the main semantic difference between the traditional word classes and the flexible word classes has to do with the values for certain meaning components. It has been demonstrated, for example, that contentives are not characterized by a positive value for the verbal feature Transitive or the nominal feature Gender (Rijkhoff 2003; Hengeveld and Rijkhoff 2005: 425-9). Presumably this is because a positive value for one of these features would immediately turn the flexible item into a verb or a noun. In short, while a contentive shares certain distributional properties with members of the four main traditional word classes, which puts it at the intersection of (i.e. inside) the category boundaries of all four traditional word classes, it also lacks some of their distinguishing semantic properties, which effectively puts it outside any of the traditional word classes. $^{8}$

(ii) Flexible items as precategorial objects Some linguists, in particular morphologists, have proposed that flexible items start out as precategorial objects and only later acquire properties that would characterize them as members of an established, traditional word class (e.g. Arad 2005; Broschart 1997; Farrell 2001; Don and van Lier this volume). It is not difficult to see that this proposal closely resembles the

\footnotetext{
8 The claim that flexible words constitute a separate category is also supported by the fact that they display so-called prototype effects (Cruse 2004: 130-1). One of the prototype effects concerns frequency of occurrence in different functions; this is discussed briefly in Section 1.3.2.2.
} 
biological process whereby totipotent or pluripotent stem cells develop into cells of a more specific type.

Note that the development of a flexible item into a functionally dedicated item should be interpreted in terms of grammatical processing. Without entering into any theory-specific details, it is generally assumed that flexible items are provided with some (verbal, nominal, adjectival, etc.) categorial specification (in the form of a syntactic slot, a label, a constructional frame, etc.) after they have been retrieved from the mental lexicon.

In sum, categorization problems in linguistics are similar to the categorization problems in other disciplines such as biology or astronomy. We find entities that cannot be properly classified, either because they would simultaneously belong to two or more traditional categories or because they are too underspecified to put them in one of the traditional categories. In the first case, we see that new categories are created, thus leading to a richer, more elaborate taxonomy of categories; in the second case, we see the postulation of a special level of precategorial or categoryneutral items.

In view of the fact that other sciences are constantly adjusting or updating their inventories in the light of new discoveries or insights, it is remarkable that some linguists are strongly opposed to revisions of the traditional system of word classification. The next section focuses on the ways in which flexible lexemes have been treated in the functional-typological literature.

\subsection{Lexical flexibility in theory and description}

\subsubsection{A brief history of lexical flexibility in the functional-typological framework}

It has long been noted that some languages do not appear to have dedicated word classes for each of the basic communicative functions PREDICATION (associated with verbs), REFERENCE (associated with nouns), and MODIFICATION (mainly associated with adjectives). ${ }^{9}$ Rather, these languages are claimed to have a word class whose members can perform more than one than one of these functions. The American structuralist Hockett (1958: 225) already discussed this cross-linguistic variation in parts-of-speech systems in terms of 'athletic squads, trained in different ways to play much the same game'. Some languages train only specialists, while others have 'all-round players'. A combination of these two types in a single language is also possible, 'producing some specialists but also good numbers of double-threat and triple-threat men'.

Hockett was not the first linguist to point out that some languages have a flexible word class. More than half a century before him, Hoffmann (1903: xx-xxi) had observed that words in Mundari (a Munda language spoken in India) display a

\footnotetext{
${ }^{9}$ For a useful overview, see Sasse (1993b: 653-61).
} 
remarkable degree of flexibility, a claim repeated in his monumental Encyclopodia Mundarica (Hoffmann and van Emelen 1928-1978: 8-9): 'Mundari words have... such a great vagueness or functional elasticity that there can be no question of distinct parts of speech in that language'. ${ }^{10}$

Especially since the 1970s, linguists working with genetically and geographically distinct languages have indicated that the traditional approach to parts-of-speech systems is rather biased towards word classes found in the familiar European languages and needs to be revised so as to be able to account for languages without distinct classes of verbs, nouns, or adjectives. Claims about the existence of flexible word classes have been made for at least the following languages and language families: ${ }^{11}$

- Malayo-Polynesian languages, e.g. Tongan (Broschart 1991, 1997; before him e.g. C. Churchward 1953: 16), Samoan (Mosel and Hovdhaugen 1992; earlier e.g. S. Churchward 1951: 126), Tagalog (Himmelmann 1991, 2008; Foley 1998), Riau Indonesian (Gil this volume and earlier publications), and Sri Lanka Malay (Nordhoff this volume and earlier publications).

- Salishan languages (Kinkade 1976; Kuipers 1968; Jelinek and Demers 1994: 718; Czaykowska-Higgins and Kinkade 1998: 35; cf. Beck this volume);

- Nootka and other languages of the Wakashan family (Sapir 1921: 133f.; Hockett 1958: 225; Mithun 1999: 378);

- Languages of the Munda family, such as Mundari (see above; cf. also Sinha 1975: 76), Santali (MacPhail 1953: 2, 9; Rau this volume) and Kharia (Peterson 2011, this volume);

- Turkic languages (Deny et al. eds. 1959; Lewis 1967/1985: 53f.; Göksel and Kerslake 2005);

- Languages spoken on the Australian continent (Dixon 1980: 271, 2002: 66; McGregor this volume).

Despite the evidence presented in these studies, the status of flexible languages remains highly controversial, among both typologists and linguistic theorists (see e.g. Ježek and Ramat 2009). This is especially true when it comes to the lack of a lexical distinction between verbs and nouns. The central position of verbs and nouns in the generative, syntactocentric framework is obvious (cf. Pinker and Bloom 1990; Baker $2003^{12}$ ), but even among many functional-typologists-who tend to be more open-minded with regard to more or less unfamiliar linguistic phenomena-the idea

\footnotetext{
10 see also Cook (1965: 108): 'The problem in Mundari morphology is that not only in syntax, but even in the morphological formation of words, a stem occurs now as one part of speech, now another.'

${ }^{11}$ See also contributions in Vogel and Comrie (eds.) (2000), Broschart and Dawuda (eds.) (2000), Ansaldo et al. (eds.) (2010).

${ }^{12}$ The generative-based theory of Distributed Morphology represents a special case in the sense that it assumes universal categories in the syntax only-not in the lexicon; cf. note 3 .
} 
of a language without nouns and verbs is not widely accepted. To get a flavour of the situation, consider the following quotations:

'Examples [of generalizations which are valid for all languages-EvL/JR] would be that languages [have] nouns and verbs (although some linguists denied even that) [...]).' (Greenberg 1986: 14)

'All languages make a distinction between nouns and verbs.' (Whaley 1997: 32)

'One of the few unrestricted universals is that all languages have nouns and verbs.' (Croft 2003: 183)

And from a recent programmatic article about language universals, on the question of whether languages can lack word class distinctions:

'Here, controversy still rages among linguists.' (Evans and Levinson 2009: 434)

To some extent, disagreement on the universality of verbs and nouns is due to different assumptions about lexical (or syntactic) categories in the various grammatical theories. In the generativist tradition, verbs and nouns are simply postulated as universal categories, whereas typologists and functionally oriented linguists (including the authors of this chapter) tend to regard verbs and nouns as language-specific categories (Croft 2001, Haspelmath 2007, Cristofaro 2009, Croft and van Lier 2012). ${ }^{13}$

Another reason why linguists do not all agree on the universality of verbs and nouns has to do with the level at which nouns and verbs are distinguished in the various theoretical frameworks. Should verbs and nouns be recognized at the level of lexical roots (i.e. in the lexicon), at the morphological level (word formation), and/or at the syntactic level (phrase structure)? This issue is discussed in some more detail in Section 1.4.

If lexical categories are language-specific, the crucial question is how they may be compared across languages. This problem has been addressed in the first systematic cross-linguistic investigation of parts-of-speech systems, carried out by Hengeveld (1992a, b), who collected information about the major lexical word classes in a representative sample of the world's languages. As he explains in the present volume, he defines word classes exclusively in terms of the functions they may serve without any additional function-indicating morphosyntactic devices (Hengeveld this volume):

'a verb $(\mathrm{V})$ is a lexeme that can be used as the head of a predicate phrase only; a noun $(\mathrm{N})$ is a lexeme that can be used as the head of a referential phrase; an adjective (A) is a lexeme that can be used as a modifier within a referential phrase; and a manner adverb (MAdv) is a lexeme that can be used as a modifier within a predicate phrase.'

13 cf. Rijkhoff (2009) on the (un)suitability of semantic categories in word order typology. On syntactic categories, see e.g. Rauh (2010). 
This method yielded the initial seven-way typology of parts-of-speech systems shown in Figure 1.1, which was gradually extended and refined in later work by Hengeveld and others (see Hengeveld et al. 2004; Smit 2001; Hengeveld and van Lier 2008, 2010; van Lier 2009).

Figure 1.1 clearly shows that there are both quantitative and qualitative differences between parts-of-speech systems of individual languages. Some languages employ a fully differentiated system, including four major lexical categories, whereas other languages may use only a hierarchically ordered subset of these four dedicated lexical categories (verb $>$ noun $>$ adjective $>$ manner adverb). ${ }^{14,15}$

Flexible languages have a major group of words that cannot be classified in terms of one of the major lexical categories, because these words can fulfil the functions that are typically served by members of two or more traditional word classes in other languages. For instance, in Samoan 'there are no lexical or grammatical constraints on why a particular word cannot be used in the one or the other function' (Mosel and Hovdhaugen 1992: 73). Since the functions referred to include predication, reference, and modification, Samoan is categorized as a language with a class of contentives in Hengeveld's classification. Turkish is an example of a language that can be analysed as displaying a flexible class of non-verbs (cf. Figure 1.1, which shows that non-verbs fulfil the functions of traditional nouns, adjectives, and manner adverbs, as illustrated in (1), (2), and (3), respectively):

Turkish (Hengeveld this volume; original examples from Göksel and Kerslake 2005: 49).

(1) güzel-im

beauty-1.Poss

'my beauty' (head of referential phrase)

(2) güzel bir köpek

beauty ART dog

'a beautiful dog' (modifier of head of referential phrase)

(3) Güzel konuş-tu- $\varnothing$

beauty speak-PST-3sG

'S/he spoke well.' (modifier of head of predicate phrase)

Dutch, finally, can be said to have a flexible class of modifiers (again, cf. Figure 1.1, which shows that modifiers fulfil the functions of traditional adjectives and manner adverbs, as illustrated in (4) and (5), respectively):

\footnotetext{
${ }^{14}$ Van Lier (2009) and Hengeveld and van Lier (2010) discuss counterexamples to the parts-of-speech hierarchy.

${ }^{15}$ The inclusion of manner adverbs in Hengeveld's hierarchy of major lexical categories is due to Dik's The Theory of Functional Grammar (Dik 1997).
} 
Dutch

(4) een mooi lied

a beautiful song

'a beautiful song' (modifier of head of referential phrase)

(5) Ze zing-t mooi

she sing-Prs.3sg beautifully

'She sings beautifully' (modifier of head of predicate phrase)

In the present volume, Hengeveld enriches his part-of-speech typology by investigating how lexical flexibility correlates with other parts of the grammar.

Hengeveld's work has been criticized by Croft $(2000,2001) .{ }^{16}$ The main difference between Hengeveld's approach and the one advocated by Croft is that the latter takes into account denotational meaning in addition to functional distribution and morphosyntactic markedness. ${ }^{17}$ Croft defines verbs, nouns, and adjectives as prototypical or unmarked combinations of a semantic class and a pragmatic function. The relevant unmarked associations of meaning and function are represented in Figure 1.2 (adapted from Croft 2001: 88):

\begin{tabular}{|c|c|c|c|c|}
\cline { 3 - 5 } \multicolumn{2}{c|}{} & \multicolumn{3}{c|}{ Pragmatic function } \\
\cline { 3 - 5 } \multicolumn{2}{c|}{} & Reference & Modification & Predication \\
\hline \multirow{3}{*}{$\begin{array}{c}\text { Semantic } \\
\text { class }\end{array}$} & Objects & unmarked noun & & \\
\cline { 2 - 5 } & Properties & & unmarked adjective & \\
\cline { 2 - 5 } & Actions & & & unmarked verb \\
\hline
\end{tabular}

FIGURE 1.2 Parts of speech as prototypical combinations of meaning and function

Croft's definition of parts of speech as typological prototypes rather than as language-specific categories renders a Hengeveld-style cross-linguistic typology of parts-of-speech systems impossible. Like Hengeveld, however, Croft's approach allows him to compare word classes across languages: the unmarked combinations of meaning and function, as represented in Figure 1.2, correlate cross-linguistically with various structural, behavioural, and semantic properties.

Croft identifies two universals of relative morphosyntactic markedness (see also Beck 2002, this volume). The first pertains to STRUCTURAL CODING, i.e. morphosyntactic markers that indicate the function in which a lexical item appears. In particular,

\footnotetext{
${ }^{16}$ For more elaborate discussion of the similarities and differences between the Hengeveldian and Croftian approaches, in the context of cross-linguistic comparability and lexical flexibility, see e.g. van Lier (2009: 33-41); Bisang (2011).

17 cf. Hengeveld and Rijkhoff (2005: 420): 'In our approach the denotation of a lexeme is completely irrelevant for its classification as a member of a specific word class. It is only the function that the lexeme fulfils in building up a predication that counts as an argument for its classification.'
} 
in any language a non-prototypical combination of meaning and function-one that departs from the situation in Figure 1.2-will be expressed with at least the same amount of structural coding as a prototypical combination.

The second universal pertains to BeHAVIOURAL POTENTIAL, a term that refers roughly to the inflectional distinctions that come with a particular function or meaning, such as tense marking for predication of actions, and number and definiteness marking for reference to objects. Croft claims that in any language a prototypical combination of meaning and function will display at least as much behavioural potential as a non-prototypical one (Croft 2001: 90, 91). ${ }^{18}$

In the context of the present volume, it must be emphasized that both of Croft's universals allow for the possibility that there is no difference in markedness with regard to the morphosyntactic encoding-in terms of structural coding and/or behavioural potential-of prototypical versus non-prototypical combinations of meaning and function. Presumably, this is what characterizes flexible word classes, as will be explained in more detail in what follows.

Another universal tendency observed by Croft relates to semantic SHIFT, i.e. the (possibly only slight) meaning change that accompanies the use of a specific word form, belonging to a certain semantic class, in a function that is not prototypically associated with this class. According to Croft, such meaning shifts will always go in the direction of the semantic class that is prototypically associated with the relevant function (Croft 2001: 73).

Croft mainly gives examples of rather substantial (and not fully predictable) shifts in meaning, such as the one between the action-meaning of a word like Tongan ako 'study' and its referential meaning 'school' (or, roughly, 'the place where one typically carries out the action-meaning), or between the object-meaning of English staple and its predicative counterpart 'to staple' (or, roughly, 'action that typically involves the referent of the object-meaning'). However, there are also more subtle types of semantic shift. Consider for instance the word $a b a$ 'person' in Teop (an Oceanic language spoken on the island of Bougainville). When this word is used in verbal (i.e. predicative) function, its meaning is 'be/was a person', as illustrated in (6). This is clearly not a prototypical action meaning, but it does express that the subject of the sentence is (or was) involved in some state of affairs, namely that of being a person. As such, the meaning shift is in accordance with Croft's prediction. ${ }^{19}$

18 Related to the issue of cross-linguistic comparability of word classes is the question of how lexical (and other linguistic) categories are represented in the speaker's mind. In general, little is known about this topic, but see Kemmerer and Eggleston (2010) for a recent overview of neurological evidence for universal and language-specific aspects of categories like nouns and verbs.

19 Cf. Rau (this volume) on the compositional semantics of Santali proper names used in predicative function. 
Teop (Mosel 2011: 4)

(6) E Magaru kou na aba vakis nana te-a taem vai ART Earthquake PTCL TAM person still IPFV.3SG PR-ART time DEM 'Earthquake was still a human being at that time.'

Conversely, when the Teop word paku 'make, do' is used in nominal (i.e. referential) function, it means '(the act of) doing/making (something)'. This is illustrated in (7), where the word describes the action 'as a unitary whole'. In Langackerian terms, this means that the action of making is scanned summariLy, like a 'thing', corresponding to a prototypical nominal meaning (and contrasting with SEQUENTIAL SCANNING, which is characteristic of verbal meanings; cf. Langacker 1987a: 72). In short, the meaning shift that a Teop action-word undergoes when used in referential function can be considered to go in the predicted direction, namely towards the objectmeaning prototypically associated with referential expressions.

Teop (Mosel 2011: 4)

(7) Eve kou to vaasusu ni bona paku sinivi 3SG PTCL REL teach APPL ART make canoe

'It was him who taught the making of the canoe.' (lit. 'the canoe making')

Apart from the very regular semantic shifts illustrated in (6) and (7), Teop also exhibits less predictable changes of the type discussed by Croft. For instance, when the Teop word beera 'big' is used in referential function it can have the meaning 'chief', presumably a lexicalized sense of 'the big one' (Mosel 2011: 4). ${ }^{20}$

Croft argues that in all cases of semantic shift-be they more or rather less regular-the precise meaning attributed to an individual word when it is used in a particular function must be stored in the mental lexicon and categorized as pertaining to that function. In other words, Croft's point is that what looks like flexibility from a purely distributional and formal perspective-characteristic of Hengeveld's approachboils down to distinct lexical classes under close scrutiny of the semantic facts.

In their target article on word classes in Mundari, Evans and Osada (2005) also argue against a flexible analysis. Beginning with Hoffmann (1903; cf. also Hoffmann and Van Emelen 1928-1978), Mundari has been put forward in several publications as a language that does not distinguish between the traditional word classes:

'[...] the same unchanged form is at the same time [...] an Adjective, [...] an Adverb, a Verb and a Noun, or to speak more precisely, it may become an Adjective etc., etc., but by itself alone it is none of them.' (Hoffmann 1903: xxi, as cited in Evans and Osada 2005: 353)

\footnotetext{
${ }^{20}$ See also Section 1.4.2 and Don and van Lier (this volume); also van Lier (2012) for discussion of different types of semantic shift in several flexible languages. On the role of metaphor and metonymy in the process of verbalization, see e.g. Heine (1997); also Hengeveld and Rijkhoff (2005: 416).
} 
However, Evans and Osada (2005: 357) claim that Mundari does have traditional word classes, but 'like all the Munda languages, makes wide use of zero conversion' (some of the problems connected with a conversion analysis are mentioned in Section 1.4.2). They put forward three (presumably necessary and sufficient) conditions for flexibility, each of which Mundari fails to meet in their view: (i) explicit semantic compositionality for argument and predicate uses, (ii) distributional equivalence, and (iii) exhaustiveness. Evans and Osada's proposal has been critically assessed in peer commentaries in the same issue of Linguistic Typology (Hengeveld and Rijkhoff 2005; Peterson 2005; Croft 2005) and are also tested in a number of contributions in the present volume (Don and van Lier; Gil; Rau; Beck-all this volume). The following section takes a closer look at the notion of lexical flexibility, in particular the way it has been characterized by Evans and Osada (2005).

\subsubsection{Establishing flexibility}

As mentioned in Section 1.3.1, three criteria have been proposed to determine whether a language has a flexible word class (Evans and Osada 2005). Each of these criteria is discussed in the following sections.

1.3.2.1 The criterion of 'explicit semantic compositionality' According to Evans and Osada's criterion of compositionality (2005: 367), 'any semantic differences between the uses of a putatively "fluid" [i.e. "flexible"-EvL/JR] lexeme in two syntactic positions (say, argument and predicate) must be attributable to the function of that position'. By this they mean to say that the semantic interpretation of a flexible lexeme must be compositionally derivable from the meaning of that lexeme plus the meaning contributed by the functional slot or constructional frame in which it is used, including any behavioural potential belonging to that function. While they admit there are some cases in Mundari that point in the direction of satisfying this criterion, they also claim that it is common for the semantic difference between argumental and predicate uses to way exceed that attributable to the syntactic position or the small perturbations due to interactions with the aspectual system'. They use the following example to illustrate their point: ${ }^{21}$

Mundari (Evans and Osada 2005: 373)
(8) a. buru=ko
bai-ke-d-a
mountain=3PL.S make-COMPL-TR-IND
'They made the mountain.'

\footnotetext{
21 The double quotation marks around mountain in (8b) are as in the original example; they serve to indicate that in Evans and Osada's view, predicative buru in (8b) is morphologically derived from buru in referential function, as in (8a).
} 


\section{b. saan=ko buru-ke-d-a \\ firewood=3PL.S "mountain"-COMPL-TR-IND \\ 'They heaped up the firewood.'}

Evans and Osada argue that there is no regular or predictable semantic correspondence between the meaning of buru in referential function in (8a) and the meaning of buru in predicative function in (8b). Therefore, they say, we must be dealing with two homophonous but distinct lexemes, each of which belongs to a traditional word class: nouns and (zero-derived) verbs, respectively.

This analysis has been challenged in Hengeveld and Rijkhoff (2005; also Hengeveld et al. 2004: 539-41; cf. also Farrell 2001), who treat buru as a flexible lexeme with a single, vague sense that is compatible with both a 'nominal' and a 'verbal' interpretation. ${ }^{22}$ In their approach, it is the actual use of a lexeme like buru in a certain function which profiles those meaning components that are relevant to that function, giving the category-neutral lexeme a particular categorial (verbal, nominal, etc.) flavour:

'By placing the flexible item in a particular syntactic slot the speaker highlights those meaning components of the flexible item that are relevant for a certain lexical (verbal, nominal, etc.) function, downplaying other meaning components.' (Hengeveld and Rijkhoff 2005: 414)

A simplified representation of this COERCION PROCESs (on coercion, see e.g. Lauwers and Willems 2011) is given in Figure 1.3 (A, B, C, etc. symbolize meaning components of Mundari buru):

\begin{tabular}{|lccc|}
\hline & A B C D E ... & Highlighted properties of buru \\
Slot: head of clause & $+\quad+\quad+\quad$ A C E $\Rightarrow$ verbal meaning ('heap up') \\
Slot: head of 'NP' & $+\quad++\quad$ B D E $\Rightarrow$ nominal meaning ('mountain') \\
\hline
\end{tabular}

FIGURE 1.3 Highlighting/downplaying of semantic components of a flexible word

Thus, whereas Evans and Osada assume that certain meaning components are added by the linguistic environment in the case of a truly flexible word (they use phrases like 'semantic increment' and 'semantic augmentation'), Hengeveld and Rijkhoff 's analysis does not involve the addition of any semantic content when a flexible word is used in actual discourse. As shown in Figure 1.3, different contexts highlight different subsets of the meaning components that are already part of the coded semantic cent of the flexible word itself (Hengeveld and Rijkhoff 2005: 415; see also Rijkhoff 2010: $238-9$ on coercion of flexible words).

\footnotetext{
22 Their analysis is based on Wilkins's (2000) account of noun semantics in the Australian language Arrernte in the context of noun classification (see also Singer 2012).
} 
A 'vague semantics analysis' is also defended by McGregor (this volume) in his account of flexibility in Gooniyandi. However, in contrast to Hengeveld and Rijkhoff, he proposes that the coded semantics generally includes only those features that are recurrent in all uses of a flexible item. All other meaning components are 'noncoded', i.e. they are not lexically stored, but rather added in compositional ways by the morphosyntactic context, or attributable to pragmatic implicatures.

Don and van Lier (this volume) also disagree with Evans and Osada's analysis, but for a different reason. They argue that a semantic shift of the type that buru undergoes in (8b) is in fact fully compositional: the specific aspectual morphology that comes along with the predicative function yields the meaning 'to become $\mathrm{X}$ ' or, in a transitive clause (as in the relevant example), 'to turn something into/make something out of X' (where X corresponds to the object-meaning of the word in question). The English translation 'to heap up' unduly suggests an idiosyncratic meaning shift. In fact, it is a periphrasis of the compositional meaning 'to turn (the firewood) into a mountain/make a mountain out of the firewood'. Thus, according to Don and van Lier examples like (8) fully satisfy the criterion of semantic compositionality. ${ }^{23}$ Likewise, Rau (this volume), argues that the interpretation of Santali proper names in predicative function is semantically compositional (Santali is also a member of the Munda family).

Example (8) involves the use of an 'object word' in predicative function. Another interesting aspect of the discussion about semantic shift relates to the reverse situation, i.e. the interpretation of 'action words' when they are used referentially. Evans and Osada assume that such a combination should yield a concrete object or person meaning, of the type 'one that X-es', where $\mathrm{X}$ corresponds to the action meaning. Considering examples such as (9), where the word dal is interpreted as an 'action nominal', they argue that this type of example involves complement clause formation.

Mundari (Evans and Osada 2005: 376)
(9) mid DaNDa dal=le
nam-ke-d-a
one stick beating=1PL.EXCL.s get-COMPL-TR-IND
'We got one stroke of beating.'

Hengeveld and Rijkhoff (2005: 420) have argued, however, that the action-nominal interpretation of words like dal in referential function is fully predictable and zeromarked (as opposed to the -ing derivation in the English equivalent). Consequently, they claim, this example meets Evans and Osada's own criterion of semantic compositionality.

\footnotetext{
${ }^{23}$ One may argue that a mountain of firewood is not a mountain in the most literal sense of feature of the landscape. In that sense, the relevance of pragmatic implicatures on the basis of world knowledge can be said to play a role in interpretation.
} 
Moreover, the action-nominal interpretation of dal is in accordance with Croft's universal directionality of semantic shift, since an action nominal describes the action 'as a whole'. As pointed out in Section 1.3.1, the semantic shift thus goes in the direction of the prototypical object meaning associated with referential expressions, even though it does not involve a literal, concrete object.

Notice, furthermore, that (in contrast to action-nominal interpretations of the type '(the act of) X-ing') the meaning 'one that X-es' requires the formation of a headless relative clause in Mundari. This is evident from the subordinator $=i q$ in example (10):

Mundari (Evans and Osada 2005: 377)

(10) susun-ta-n=iq

dance-PROG_OR-INTR=3SG.S

'the one who is dancing'

This finding ties in with Beck's analysis of Lushootseed (this volume) as a language displaying UNidiReCTIONAL flexibility (see also Section 1.3.2.2), where semantic 'nouns', i.e. object or person words, can be used predicatively as 'verbs', but not vice versa. In Lushootseed, as in Mundari, action words must be turned into headless relative clauses in order to obtain a 'one that X-es' meaning. However, in Lushootseed the zero-marked use of action words in referential function with the meaning '(the act of) X-ing' is ruled out; this meaning can only be obtained by means of overt derivation, similar to English -ing formation.

1.3.2.2 The criterion of 'bidirectional distributional equivalence' Evans and Osada's second criterion requires that truly flexible lexemes are characterized by distributional equivalence that is fully bidirectional, i.e. members of two putative classes (object-denoting and action-denoting lexemes) must be equally acceptable in the functions of argument and predicate (Evans and Osada 2005: 366-7). There are two aspects of this criterion that deserve some further attention. First, one could ask whether 'equally acceptable' should imply full symmetry with respect to frequency of occurrence in any given function in natural discourse. In our view this is not a reasonable requirement. In all languages we find that certain meanings are more strongly connected with a particular function than other meanings, whatever the nature of the parts-of-speech system. In fact, this is what one should expect to find in all natural languages as one of the prototype effects in the area of word categorization (Croft 2001; Cruse 2004: 130-1). For example, most English adjectives can occur as attributes and predicates, though usually not with the same frequency. ${ }^{24}$ The following example from Merriam Webster's Dictionary of English Usage (1994: $373,617)$ illustrates: ' $[\ldots]$ the adjective magic is almost exclusively an attributive

\footnotetext{
${ }^{24}$ Recall that, according to Hengeveld's definitions (Section 1.3.1), a true noun, adjective, or manner adverb may also serve as the sentence predicate.
} 
adjective [...]. Magical can be either an attributive or a predicate adjective, but attributive uses are about three times as common as the others.'

Similar things can be said about the frequency of use of members of a flexible word class. In a corpus-based study of Teop (also mentioned in Section 1.3.1), Mosel (2011: 5) shows that paku 'do, make' appears 859 times in predicative function against 11 times in referential function. ${ }^{25}$ Conversely, taba 'thing' occurs 465 times in referential function and only 5 times in predicative function. This shows that words with mainly action or object semantics have a (more or less strong) preference for the predicative or the referential function, respectively, but are nonetheless fully grammatical in both these functions. Interestingly, Teop words that have neither action nor object semantics, such as kikis 'strong' and mararae 'happy', are more evenly distributed over various functions: kikis, for instance, is used 15 times as a predicate, 11 times in referential function, and 18 times as a modifier. In other words, flexible words can be ranked according to frequency of occurrence in different functions, just like members of traditional, non-flexible word classes.

These data suggest that the infrequency of certain combinations of meaning and function may also lead to accidental gaps. In fact, Evans and Osada (2005: 381) claim that certain Mundari kinship terms, such as misi ('sister') cannot be used in predicative function, but Peterson (2005) has argued that no such restriction exists, and that it is rather a matter of finding a suitable pragmatic context, as illustrated by elicited example (11):

Mundari (Peterson 2005: 401)

(11) ini? an-a? misi-w-a-?e

3SG 1SG-GEN sister-W-IND-3SG

'She became my sister (e.g. when my father remarried to a woman who had a daughter).'

Statements to the same effect can be found in Mosel and Hovdhaugen's Samoan Reference Grammar (1992: 77) in their discussion of the 'verbal' and 'nominal' use of words ('roots') in actual discourse:

'Not all roots occur with the same frequency as verbs and nouns. Some roots predominantly function as verbs, whereas others are more likely to be found in the function of nouns. Until now we have not, for instance, found alu 'go' in a nominal function or mea 'thing' in a verbal function [...]. But we hesitate to say that alu is inherently a verb and mea inherently a noun for two reasons. Firstly, we cannot find any functional explanation why alu should not be used as a noun and mea as a verb, whereas, for instance, gaoi 'thief, to steal' and tagata 'person, to be a person' are bi-functional. And, secondly, previous experience taught us to be careful with

\footnotetext{
${ }^{25}$ Note that Mosel (2011) does not analyse Teop thing/person and action words as a single flexible class, as there are certain distributional differences to be found between the two types of words, specifically in constructions where they are modified by a property word.
} 
classifications. The more texts we analysed, and included in our corpus, the more items were unexpectedly found in nominal or verbal function. ${ }^{26}$

This indicates that Evans and Osada's criterion of distributional equivalence is too strictly formulated. Flexible words are not attested in all functions in the same frequency, but they have the possibility of occurring in the various functions, given a suitable pragmatic context.

A second aspect of the criterion of distributional equivalence crucially involves the notion of BIDIRECTIONALITy. ${ }^{27}$ This means that in order 'to establish that there is just a single [flexible-EvL/JR] word class, it is not enough for Xs to be usable as Ys without modification: it must also be the case that Ys are usable as Xs' (Evans and Osada 2005: 375). As discussed in Section 1.3.2.1, the use and interpretation of Mundari action and object words in referential and predicative function, respectively, is subject to controversy. With regard to the predicative use of object words, Evans and Osada point out that in Mundari the compositional semantic interpretation 'be $\mathrm{X}$ ' (where X corresponds to the object-/person-meaning of the lexeme, cf. example (6)) is obtained only in combination with a copula (see example (12a)). Without a copula, they claim, the construction receives an idiosyncratic interpretation (example (12b)). This would violate the criterion of compositional semantics, hence (12b) cannot be regarded as an instance of flexibility, according to Evans and Osada.

Mundari (Evans and Osada 2005: 372)

(12) a. ne dasi hoRo tan-iq
this servant Munda COP-3SG.s
'This servant is a Munda.'
b. ne dasi hoRo- $a=e q$
this servant Munda-IND=3sG
'This servant speaks Munda.'

This analysis has been criticized in peer commentaries of Evans and Osada's target article on the Mundari part of speech system. Peterson (2005: 396) points out that Kharia (also a member of the Munda family) only uses a copula in non-dynamic sentences. He furthermore indicates that the predicative use of 'nominal' phrases without a copula is more widespread in Mundari than is suggested in Evans and Osada's article. Similarly, Rau (this volume) shows that in Santali, another Munda language, copulas only appear in certain aspectual contexts. Finally, Hengeveld and Rijkhoff (2005: 421) point out that Evans and Osada fail to distinguish between the predicative use of a (bare) lexeme and the predicative use of phrasal constructions.

\footnotetext{
${ }^{26}$ Hengeveld and Rijkhoff (2005: 412) have pointed out that Mosel and Hovdhaugen appear to be unaware of the fact that they actually provided an example of $a l u$ in nominal function some pages earlier.

27 See Beck (this volume) for an analysis of unidirectional flexibility.
} 
Since the example with the copula (12a) does not involve a lexeme in predicative function but rather a member of a phrasal category, it does not prove that Mundari words are not flexible.

1.3.2.3 The criterion of 'exhaustiveness' The third and final criterion concerns exhaustiveness of flexibility, which Evans and Osada (2005:378) formulate as follows:

'It is not sufficient to find a few choice examples which suggest word class flexibility. Since word classes are partitionings of the entire lexicon, equivalent statements need to hold for all relevant words in the lexicon that are claimed to have the same class.'

While at first blush this seems a reasonable requirement, which has actually been proposed by other scholars as well (see e.g. Baker 2003: 117; Hengeveld et al. 2004: $538^{28}$ ), there are at least two methodological problems connected with this criterion.

First, the criterion is obviously hard to assess, since in practice it is not feasible to check all the lexemes of a language (see Gil this volume for discussion and application of the criterion to Riau Indonesian). Therefore, Evans and Osada pursue a quantificational approximation of the degree of lexical flexibility in Mundari through random sampling, in combination with more controlled assessments of the distributional possibilities of various semantic lexeme classes. Temporarily leaving aside the issue of semantic shift, they arrive at a characterization of the Mundari situation as comparable to English: using the same lexeme as a noun and a verb is rather common, but not unconstrained.

At a more detailed level, Evans and Osada argue that specific semantic subclasses-such as proper names, certain kinship terms, and most animal and plant names-do not occur as predicates. Apart from the possibility of accidental gaps in the data due to frequency asymmetries (see Section 1.3.2.2), this brings up the role of subclasses in word categorization. The problem associated with subclasses is recognized in many studies on word classes, here illustrated with a quotation taken from Schachter and Shopen (2007: 4; emphasis in the original):

'It must be acknowledged [...] that there is not always a clear basis for deciding whether two distinguishable open classes of words that occur in a language should be identified as different parts of speech or as subclasses of a single part of speech. The reason for this is that the open parts of speech classes must be distinguished from one another on the basis of a cluster of properties $[\ldots]$. Typically there is some overlap, some sharing of features, as well as some differentiation.'

Croft's theory of parts of speech, outlined in Section 1.3.1, has the advantage of getting around the problem of subcategorization, without falling prey to what Croft

\footnotetext{
${ }^{28}$ But cf. Luuk (2010: 362), who argues that a language with a single lexeme that can be used as a verb and a noun can be regarded as displaying a flexible category. This leads him to question whether there are languages that do not have a class of 'flexibles'.
} 
describes as 'methodological opportunism', i.e. defining parts of speech on the basis of an essentially randomly selected set of distributional properties (Croft 2005: 434, see also Croft 2000, 2001). Smith (2010) criticizes Croft's approach as being unable to define word classes in individual languages. However, as Croft (2010: 791) states in his reply, this is not the intention of his theory; for the formulation of Croft's universals of relative markedness and semantic shifts it is simply irrelevant to decide whether a certain group of lexemes counts as a 'major word class' or as a 'sub-class of a major word class'.

\subsubsection{Summary}

In this section we have reviewed some key issues in the discussion concerning flexible word classes. While claims of lexical flexibility date back more than a century, and while flexible languages have a place in some current functional-typological approaches to parts-of-speech systems, there appear to be deep-rooted differences regarding the criteria for lexical flexibility and how they should be employed in the analysis of linguistic forms and constructions.

The next section puts flexibility in the larger context of the grammatical system. First, we discuss the notion of CONVERsion as a lexical or syntactic phenomenon. Subsequently, we review a number of recent studies that explicitly correlate flexible behaviour of various kinds of linguistic forms and constructions (i.e. not just with regard to lexemes, but also phrases and clauses) with categorial specificity at other grammatical levels.

\subsection{Flexibility beyond the lexicon}

\subsubsection{Flexibility and levels of grammatical analysis}

As mentioned in the previous section, some of the disagreements concerning flexible word classes reside in differences with regard to the grammatical level at which categorial distinctions are deemed to be present or absent: in the lexicon, at the morphology level, and/or at the level of syntactic representation. What the various approaches share is that flexibility is only claimed in the absence of any overt formal marking of (re)categorization, a phenomenon sometimes referred to as CONVERSION. Section 1.4.2 gives an overview of such zero-marked (re)categorization at various levels of analysis.

Recently, a number of studies (also in the present volume; see in particular the contributions by Hengeveld, Gil, and Bisang) have explicitly addressed the issue of multiple-level flexibility. In particular, these studies suggest that there may be certain constraints on the degree of flexibility displayed at different levels in the grammar, such that multifunctionality at one level requires counterbalancing by functional specificity at another level, in order to maintain the overall functional transparency in 
the grammar of a language. We review these studies in Section 1.4.3, which also sketches a way to extend the notion of flexibility to grammatical material like affixes and particles.

\subsubsection{Zero-marked (re)categorization: approaches to conversion}

Since a full account of the notion of conversion is beyond the scope of this introductory chapter, this section only outlines the various perspectives on this phenomenon that have been offered in the literature. Conversion is usually defined as a process that relates two formally identical but categorially distinct words (Balteiro 2007, Bauer and Valera Hernández 2005, Bauer 2008). Within this general definition, however, a number of different approaches can be distinguished. An important distinction is often made between (i) approaches that view conversion as a lexical phenomenon, i.e. as a process that produces new lexical items without overt derivational marking, and (ii) approaches that consider it to be primarily a syntactic phenomenon, involving the zero-marked use of a lexeme in multiple syntactic contexts.

The lexical approach can be further divided into CONVERSION and ZERO-DERIVATION. The former term is usually reserved for approaches that view the relation between the two members of a pair of formally identical but categorially distinct word forms as resulting from an identity operation on one member, producing the other member as its output. Crucially, this type of operation does not assume the presence of a zero-affix (see e.g. Beck this volume). ${ }^{29}$

This is different, as the term implies, in the case of zero-derivation, where the two members of a pair are assumed to be related through zero-affixation. Some have argued that the postulation of a zero-morpheme is plausible only if it contrasts with an overt derivational morpheme that carries out the same operation; the co-called 'overt analogue criterion' (Sanders 1988). While this criterion has the advantage of providing a formal solution to the obvious testability problem associated with invisible morphemes, it also raises problems of its own (as Sanders himself acknowledges). For one thing, the criterion implies that zero-derivation is somehow the unexpected option, while overt derivation would be the expected or 'standard' situation. More importantly, in some cases there is no overt analogue, while at the same time there is semantic and functional evidence for a category-changing operation. Some scholars would claim that in such cases 'meaning' is simply not accompanied by (phonological) 'form', independently of any contrast with an overt morpheme (e.g. Don 1993; Don and van Lier this volume).

In discussions about conversion and zero-derivation it is often assumed that lexemes belong to one of the traditional word classes, (tacitly) conceived of either

\footnotetext{
${ }^{29}$ Sometimes, this particular approach to conversion is considered distinct from so-called relisting (Lieber 1981), where the two members are analysed as semantically related but separate lexical entries. Conversion (in this narrow sense) and relisting have in common that no zero-affixes are postulated.
} 
as universal or as language-specific categories (Don 2005, Bauer 2008). However, rather than assuming that one form is zero-derived from the other, or that one form serves as the input of the conversion process and the other one as the output, it is also possible that both forms are output forms, which are symmetrically related to an uncategorized root (Luuk 2010: 352; cf. Don and van Lier this volume and references therein). Still, scholars differ in whether they view the derivation of categorially specified output forms from roots as a lexical or a syntactic process, or allow for both options. In all cases, however, the key argument in favour of flexible, uncategorized, or category-neutral roots is that it is the most parsimonious option: if there is no formal evidence for a change in word class, this is because there is no such change in the first place (Farrell 2001, Luuk 2010, Peterson this volume, Gil this volume). ${ }^{30}$

In the absence of any overt formal marking, the type of semantic shift accompanying the use of a lexeme in a particular syntactic function plays an important role in the various analyses that have been proposed. Recalling the discussion of semantic compositionality in Section 1.3.2.1, we may distinguish a number of different positions.

One possibility is to consider all semantic shifts, be they regular or irregular, as stored in the mental lexicon and labelled (i.e. categorized) for a specific syntactic function. 'Flexible' words are then merely pairs of separate, homonymous lexemes, which are semantically related through lexical conversion (Croft 2005, Beck this volume).

Another position would maintain a contrast between compositional and noncompositional semantic shifts, with only the former type taken as evidence for specialized lexical categories. As mentioned above, while some would assume that lexemes are stored in the lexicon with their specific categorial value and can be related through lexical conversion or zero-derivation (e.g. Evans and Osada 2005), others propose flexible or uncategorized roots from which categorially differentiated lexemes are zero-derived (e.g. Don and van Lier this volume). Either way, this position holds that compositional semantic shifts result from the categorial (further) specification of a root or lexeme through the syntactic function in which it is used. The meaning of a flexible lexeme or root is considered to be fixed and the semantic shift is added by the morphosyntactic context (Evans and Osada 2005; Don and van Lier this volume; Rau this volume; but note that they do not necessarily agree on what counts as compositional meaning shift).

Finally, some scholars do not consider any type of semantic shift as evidence for (re)categorization through zero-derivation or conversion: flexible lexemes are treated as monosemous elements. This view is advocated by Hengeveld and Rijkhoff (2005) and McGregor (this volume). They differ, however, in their assumptions about the

\footnotetext{
30 see also Barner and Bale (2002) for psycholinguistic arguments (related to language acquisition and category-specific neurological deficits) in favour of a theory that assumes uncategorized lexical roots.
} 
semantics of flexible lexemes. While Hengeveld and Rijkhoff hold that different contexts highlight different subsets of the total set of meaning components of a lexeme ('coercion'; see Section 1.3.2.1), McGregor argues that the stored meaning of a flexible lexeme consists only of those meaning components that are stable across its various functions.

The different approaches to conversion, particularly with regard to semantic shift, indicate that flexibility is not necessarily just a matter of lexical categorization. Irrespective of the details of the analyses, they all suggest that lexical flexibility is a phenomenon that should be considered in close connection with morphology and syntax, i.e. in the larger context of the grammatical system of a language. The next subsection further explores flexibility from this wider perspective.

\subsubsection{Flexibility and functional transparency}

So far we have only been concerned with lexical flexibility, but it has been argued in several recent studies that larger linguistic units, such as phrases and clauses, can also display flexible behaviour. In the present volume such cases of flexibility are considered in connection with the following languages: Riau Indonesian, Kharia, Santali, Samoan, Tagalog, and Lushootseed (see also van Lier 2009). The studies in question indicate that there are intricate relations between the degree of flexibility displayed by various construction types and the level of grammatical complexity of the construction. For example, it has been shown that the categorial specificity of linguistic units increases-resulting in a decrease in flexibility-when they become structurally more complex. Thus, Haig (2006: 45) (see also Lehmann 2008) distinguishes between the successive complexity levels of (i) roots ('radical elements'), (ii) input of derivation (i.e. the extent to which derivational processes select specific base categories; cf. Bisang this volume), (iii) output of derivation, and (iv) inflectional morphology. $\mathrm{He}$ coined the 'principle of successively increasing categorization', stating that categorial distinctions will increase and/or become more fine-grained as one moves up these levels.

Haig's generalization is intimately related to the idea that multifunctionality or flexibility at one level of the grammatical system must be counterbalanced by categorial specificity at another level in order to guarantee FUNCTIONAL TRANSPAR$\mathrm{ENCY}$, i.e. the functional identifiability of linguistic units at the level of an actual utterance. This idea is formulated as follows in Frajyzngier and Shay (2003: 4-5) (see also Lehmann 2008: 557; Hengeveld et al. 2004; Hengeveld this volume; cf. Gil this volume; Bisang this volume):

'Every constituent must have a transparent function within the utterance. [...] This is achieved either through the inherent properties of lexical items in the clause or through the system of grammatical means, which include affixes, adpositions, linear order and free morphemes with grammatical functions.' 
In line with the trend to put flexibility in a larger grammatical context, we would finally like to outline a proposal for a typology of linguistic flexibility that includes both lexical (L) and grammatical (G) material. In the case of grammatical material, the notion FLEXIBLE refers to the fact that the same grammatical marker (expressing the same semantic notion) can appear both in clauses and in referential phrases (Rijkhoff 2008b). For example, in Fongbe (a Kwa language spoken mainly in Benin) the same morpheme is used to express definiteness in the referential phrase and realis in the clause. In either case the morpheme indicates that the entity in question, i.e. the crab or John's arrival, (already) has an identifiable place (is 'grounded') in the world of discourse at the moment of speaking (see Rijkhoff and Seibt 2005: 102; DET = determiner-glosses as in the original):

Fongbe (Lefebvre 1998: 94, 99; see also Lefebvre and Brousseau 2002)

(13) $N$ dú àsón j́

I eat crab DET

'I ate the crab (in question/that we know of).'

(14) Jan wá j́

John arrive DET

'Actually, John arrived.'

In the following example from Jacaltec (a Mayan language spoken in Guatemala), the same morpheme expresses nonspecific-indefiniteness in the referential phrase and irrealis in the clause. Here the morpheme indicates that the entity (the sleeping event, the pot) does Nот have an identifiable location in the discourse world at the moment of speaking (variation between $-o j$ and $-u j$ is due to vowel harmony): $:^{31}$

Jacaltec (Craig 1977: 93)

$\begin{array}{llllll}\text { (15) } \quad \text { X- } \varnothing \text {-'oc } & \text { heb } & \text { ix } & \text { say-a' } & \text { hun-uj } & \text { munlabel } \\ \text { AsP-ABs.3-start } & \text { PL } & \text { woman } & \text { look_for-FUT } & \text { a-oJ } & \text { pot }\end{array}$

'The women started looking for a pot.' [- $u j$ indicates NONSPECIFIC reference]

(16) Way-oj ab naj sleep-oJ EXH CLs/he

'Would that he slept!' [-oj indicates IRREALIS/EXHORTATIVE mood]

The two parameters (lexical vs grammatical and flexible vs rigid) give us four possible combinations or 'language types' (obviously each 'type' is an idealization):

\footnotetext{
31 Other languages displaying a certain degree of grammatical flexibility are, for example: Rapanui (Du Feu 1987, 1989, 1996), Koasati (Kimball 1991: 405). Nordlinger and Sadler (2004) discuss aspect/tense/mood marking on referential phrases.
} 


\section{(17) Typology of Flexibility}

1. $\mathrm{L}_{\mathrm{FLexible}} / \mathrm{G}_{\mathrm{FLexibLE}}$ : Languages with flexible lexemes and flexible grammatical markers that can occur both in clauses and in referential phrases;

2. $\mathrm{L}_{\mathrm{FLEXIBLE}} / \mathrm{G}_{\mathrm{RIGID}}$ : Languages with flexible lexemes and rigid grammatical markers that occur either in clauses or in referential phrases;

3. $L_{\text {RIGID }} / G_{\text {FLeXIBLE }}$ : Languages with rigid lexemes and flexible grammatical markers that can occur both in clauses and in referential phrases;

4. $\mathrm{L}_{\mathrm{RIGID}} / \mathrm{G}_{\mathrm{RIGID}}$ : Languages with rigid lexemes and rigid grammatical markers that occur either in clauses or in referential phrases.

Languages of Type 1 ( $\mathrm{L}_{\text {FLexible }} / \mathrm{G}_{\text {FLexible }}$ ), which would be characterized by flexible lexemes as well as flexible grammatical morphemes, should be extremely rare or even non-existent, since such 'double' flexibility would seriously interfere with successful and efficient communication. In other words, languages of Type 1 would not be functionally transparent (see above) or ECONOMICAL. Languages of Type 2 ( $\mathrm{L}_{\text {FLEXIBLE }} /$ $\left.\mathrm{G}_{\text {RIGID }}\right)$ and Type $3\left(\mathrm{~L}_{\text {RIGID }} / \mathrm{G}_{\text {FLEXIBLE }}\right)$, which display flexibility in one domain and rigidity in the other, should be frequently attested, since they have the double functional advantage of combining transparency with economy. Type $4\left(\mathrm{~L}_{\text {RIGID }} /\right.$ $\mathrm{G}_{\mathrm{RIGID}}$ ), finally, is also functionally transparent, but not the most economical, since it displays redundant categorial specificity. Notice, however, that Hengeveld et al. (2004) have shown that redundant categorial marking in the form of rigid word classes in combination with rigid word order is cross-linguistically not uncommon.

Obviously, this typology needs to be refined, also taking into account, for example, different degrees of flexibility and rigidity (cf. Bisang this volume, who investigates several languages in terms of the typology proposed here). Notice, furthermore, that this four-way typology largely ignores the diachronic dimension, in particular the role of metaphor and metonymy in the (further) grammaticalization of grammatical markers leading to grammatical flexibility as described above, as when the same grammatical marker can occur in clauses and referential phrases (Rijkhoff and Seibt 2005). For example, it has been argued that the Indo-European collective marker on nouns (currently still visible in e.g. German Ge-brüder as in Gebrüder Müller 'Müller Brothers' and Ge-birge 'mountain range') has developed into a marker of perfectivity in the Germanic, Slavic, and other Indo-European languages (von Garnier 1909, Rijkhoff 2008b: 29-32). ${ }^{32}$ In some Germanic languages, the element became ultimately associated with the past participle form of the verb, as in German ge-sprochen 'spoken' (Kirk 1923: 65).

\footnotetext{
32 See Leonid Kulikov's message to the Discussion List for The Association for Linguistic Typology, 31 March 1998 (Subject: collective/perfective). The message can be found at $<$ http://listserv.linguistlist.org/cgi$\mathrm{bin} /$ wa $? \mathrm{~A} 2=$ ind $9803 \mathrm{e} \& \mathrm{~L}=$ lingtyp $\& \mathrm{D}=1 \& \mathrm{~F}=\& \mathrm{~S}=\& \mathrm{P}=606)>$.
} 


\subsubsection{Summary}

In this section we have characterized flexibility as a phenomenon that may apply in different degrees to different levels of the grammar of an individual language. In particular, certain groups of language-specific linguistic items (including lexical roots, morphologically derived words, and syntactic elements) display a certain amount of multifunctionality or categorial specificity. Flexibility and rigidity are thus not mutually exclusive notions, but rather seem to complement each other in the grammatical system of a language. Exactly which groups of linguistic elements display which categorial values in a particular language is a matter of formal and semantic markedness criteria.

\subsection{This volume}

This final section gives a brief outline of the other contributions to the present volume.

Kees Hengeveld-Parts-of-speech systems as a basic typological determinant

This contribution provides an overview of evidence, accumulated in typological research carried out by Kees Hengeveld and his colleagues over the past two decades, showing that extensive flexibility at the lexical level correlates with a number of properties at various other levels of grammatical organization.

In particular, Hengeveld shows that languages with highly flexible lexemes ensure the functional identifiability of this lexical material through rigid marking of syntactic slots. This ties in with the observation that these languages allow not only single lexical items to appear in multiple functions, but also more complex phrasal and clausal constituents. In addition, Hengeveld shows that lexemes that are flexible in their functional distribution must be stable in other respects: they are not divided into form-based (morphologically or phonologically conditioned) or meaning-based subclasses, and there are also no irregular or suppletive forms of individual lexical items.

Emerging from the conglomerate of data presented by Hengeveld is a structural profile typical for languages with flexible word classes. The pervasiveness of this profile correlates with the specific degree of flexibility displayed by a particular language's lexical organization.

Jan Don and Eva van Lier-Derivation and categorization in flexible and differentiated languages

Jan Don and Eva van Lier focus on Evans and Osada's criterion of compositionality: What is the semantic interpretation of formally identical lexical forms used in different syntactic contexts, and what does this imply in terms of the presence or absence of categorial distinctions in the lexicon of specific languages? 
Don and van Lier compare descriptive data of three prominent candidates for flexible languages-Kharia (Munda, India), Tagalog (Malayo-Polynesian, Philippines), and Samoan (Oceanic, Samoa) - with Dutch, the authors' native language, which displays distinct lexical classes of verbs and nouns. The data show that compositional and non-compositional semantic shifts are attested in all four languages.

The crucial difference between 'flexible' and 'differentiated' languages, according to Don and van Lier, is that the latter typically combine lexical and syntactic categorization into a single operation, while in the former language type they are separated. More specifically, while in differentiated languages roots must combine with a categorial label before they can be further processed by the morphology and syntax, flexible languages can (zero-)derive and combine roots without affecting their distributional freedom. Categorization takes place only at the level of syntactic slots.

The proposal by Don and van Lier adopts Peterson's analysis of Kharia (as presented in this volume and previous publications; see e.g. Peterson 2005, 2011), and is also broadly in line with Gil's analysis of Riau Indonesian, Nordhoff's analysis of Sri Lanka Malay, Rau's analysis of Santali, and Bisang's analysis of Late Archaic Chinese (all this volume). In all these languages that are claimed to display a class of flexible lexemes, the interpretation of lexical material at the syntactic level is shown to be semantically compositional. In addition to this, there may be non-compositional shifts, accompanying non-categorizing (zero-) derivational processes.

\section{David Gil-Riau Indonesian: a language without nouns and verbs}

Gil's paper starts out with a general assessment of the way in which flexible parts of speech are often approached in the (descriptive and theoretical) literature. Indeed, he argues, the term 'flexibility' in itself suggests a departure from the assumed default scenario: the presence of distinct lexical categories, in particular of nouns and verbs. Comparing these with a category like dual number, Gil emphasizes that we should assume a newly-to-be-described language not to have nouns and verbs until we find evidence to the contrary, rather than following the reverse practice.

The empirical contribution of Gil's work is an analysis of Riau Indonesian (Malayic, Sumatra), consisting of two parts. Firstly, he shows that there are no differences in the behaviour of object-denoting and action-denoting lexical items, with regard to their ability to be used in a set of ten different syntactic construction types. Secondly, Gil demonstrates that Riau Indonesian meets the three criteria for flexibility as proposed by Evans and Osada (2005): compositionality, bidirectionality, and exhaustiveness. To assess the latter criterion Gil randomly selects a set of content words and tests their acceptability in a number of different syntactic constellations.

Gil rounds off with a typological proposal, which views flexibility as a scalar phenomenon that may apply independently to the levels of morphology, syntax, 
and semantics. This perspective is in accordance with other proposals expressed in recent literature on flexibility (discussed in the previous section), including our own proposal (Section 1.4.3) and other contributions to the present volume.

\section{John Peterson-Parts of Speech in Kharia: a formal account}

This is the first of two case studies on Munda languages, i.e. languages that are closely related to Mundari (see Section 1.3).

The first part of John Peterson's paper is devoted to a general introduction to Kharia (South Munda, India), demonstrating that any combination of lexical material (be it in the form of single items or complex phrases) can be used in predicative, referential, and attributive functions, with compositional semantic results.

In the second part of the paper, Peterson provides a monostratal theoretical account of the Kharia data in terms of two structural categories: TAM/Personsyntagmas and Case-syntagmas. The former consist of a lexical or phrasal 'content head' in combination with enclitic markers for TAM and Person, while the latter involve a combination of a 'content head' with a case marker.

Peterson shows that formally analysing Kharia as a language without lexical categories of nouns and verbs is not only possible, but also the most economic, adequate, and theory-neutral way of describing this language.

\section{Felix Rau-Proper names, predicates, and the parts-of-speech system of Santali}

Felix Rau's analysis of Santali starts with a brief outline of the formal properties of predicates and arguments in this language. In the remainder of the paper he concentrates on the predicative function, showing that virtually every lexeme can fulfil this function, taking the same set of inflectional distinctions, and receiving compositional semantic interpretations. As a specific test case for this claim, Rau then examines the acceptability of proper names in predicate function. While in some context these items, whose semantics strongly tend towards the referential function, need copula support to be used as main predicates, there are other contexts in which they behave flexibly and compositionally.

The case of proper names in Santali is especially interesting in view of Hengeveld and Rijkhoff's (2005) critique of the Mundari data as presented by Evans and Osada (2005). As mentioned in Section 1.3.2.3, Hengeveld and Rijkhoff argue that Mundari uses a copula with phrasal predicates, but not with bare object-denoting lexemes, presumably because the latter are typically used in property-assigning contexts.

David Beck-Unidirectional flexibility and the noun-verb distinction in Lushootseed David Beck's contribution is unique in the present volume in arguing explicitly in favour of a universal noun-verb distinction. In his view, such a distinction is present not only in the particular language analysed in this paper, Lushootseed (a moribund 
Salishan language of Washington State), but also in languages such as Tongan, which have been proposed as candidates for 'true' flexibility (see Broschart 1997). Following Evans and Osada (2005) and Croft (2000, 2001, 2005), Beck argues that semantic shifts associated with the use of formally identical lexemes in different functions, while often non-arbitrary or semi-systematic, are nevertheless not predictable and must be memorized by speakers as separate, categorially specified items.

In specific relation to Lushootseed, Beck shows that the noun-verb distinction in this language is effectively neutralized in only a single functional context, namely predication. This means that Lushootseed does not satisfy Evans and Osada's criterion of equivalent combinatorics: while object words can be used as predicates, action words can function as arguments only if they take the form of headless relative clauses or overtly derived action nominals. At the same time, Beck's careful argumentation takes issue with the long-standing idea that Salish languages are 'omni-predicative', meaning that arguments would always take the form of verbal expressions in these languages.

William B. McGregor-Lexical categories in Gooniyandi, Kimberley, Western Australia

William McGregor's paper on the Western Australian language Gooniyandi provides another illustration of the intricate distributional details that descriptive linguists are faced with when describing the parts-of-speech system of an individual language. Moreover, this case study broadens the range of types of lexical flexibility comprised in the present volume: unlike languages with a single flexible class of content lexemes (e.g. Riau Indonesian, Kharia), Gooniyandi displays a class of flexible object- and property-denoting lexemes, which can be used as heads and modifiers in referential expressions (cf. Hengeveld and van Lier 2008, 2010; van Lier 2009).

McGregor considers the theoretical implications of the Gooniyandi data from various perspectives. Firstly, he compares the frameworks of Functional Discourse Grammar and Semiotic Grammar, taking into account the problem of cross-linguistic comparability of word classes. A second point of interest, mentioned already in Sections 1.3.2.1 and 1.3.3, concerns McGregor's assessment of the semantics of Gooniyandi flexible lexemes. In particular, he proposes a vagueness analysis that is fully compatible with compositional se tic shift, and as such differs from the one advocated by Rijkhoff and Hengeveld (2005) (see also Hengeveld et al. 2004).

\section{Sebastian Nordhoff_Jack of all trades: the Sri Lanka Malay flexible adjective}

Sebastian Nordhoff demonstrates that the presence of a class of fully flexible lexemes does not preclude the presence of functionally specialized classes of nouns and verbs. In particular, this study shows that Sri Lanka Malay has nouns, verbs, and a class that Nordhoff calls Flexible Adjectives-the latter containing property-denoting lexemes, 
which are usable not only as modifiers, but also as predicative and referential expressions. As Nordhoff points out, this analysis ties in neatly with recent work on the Hengeveldian typology of parts-of-speech systems, which accounts for a range of cross-linguistic variation that is wider than in earlier work (Hengeveld and van Lier 2008; see also van Lier 2009 and Hengeveld and van Lier 2010).

Another important focus of Nordhoff's paper concerns his diachronic account of the development of the Sri Lanka Malay word class system, which he shows to be codetermined by a combination of external and internal factors: language contact with Sinhala and Tamil on the one hand, and the optional application of categorizing derivational morphology on the other hand.

Walter Bisang-Word class systems between flexibility and rigidity: an integrative approach

The diachronic development of part of speech systems and the influence of language contact on the course of that development are also emphasized by Walter Bisang. His contribution takes as its point of departure the typology of flexibility presented in Section 1.4.3. Bisang explores the empirical limits of this typology by means of case studies of Late Archaic Chinese, Khmer, Classical Nahuatl, and Tagalog.

For the first language, he shows that there is no principled distributional difference between object- and action-denoting lexemes, and that the interpretation of these lexemes in non-prototypical functions is semantically regular. Bisang proposes that the Late Archaic Chinese system, in which the function of lexemes is signalled solely by the syntactic slots in which they appear, developed through the loss of morphological devices that were used in an earlier stage of the language.

For Khmer, it is shown that while derivational morphology in this language is flexible to the extent that the same morpheme can be used to derive verbs and nouns, the output forms involving individual lexical bases are clearly categorized as noun or verb, with semantic interpretations that range from very systematic to rather idiosyncratic. Bisang suggests that the Khmer derivational morphemes have not undergone further functional specialization due to the adoption of alternatives strategies from Thai and other East and mainland South East Asian languages.

More evidence that flexibility can - and should-be attributed to specific grammatical subsystems comes from the analyses of Nahuatl and Tagalog. In particular, while both languages have just a single word class for the purposes of syntactic distribution (verbal or nominal), distinct lexical categories are relevant at the level of inflectional and derivational morphology. 